\title{
Prevalence of Patient-Reported Social Risk Factors and Receipt of Assistance in Federally Funded Health Centers
}

\author{
Emilia De Marchis, $M D, M A S^{7}$, Matthew Pantell, $M D M S^{2}$, Caroline Fichtenberg, $P h D, M S^{3}$, \\ and Laura M. Gottlieb, MD MPH ${ }^{1,3}$
}

'Department of Family \& Community Medicine, University of California, San Francisco, San Francisco, CA, USA; ${ }^{2}$ Department of Pediatrics, University of California, San Francisco, San Francisco, USA; ${ }^{3}$ Social Interventions Research and Evaluation Network, University of California, San Francisco, San Francisco, USA.

J Gen Intern Med 35(1):360-4

DOI: 10.1007/s11606-019-05393-w

(c) Society of General Internal Medicine 2019

\section{INTRODUCTION}

Interest in integrating social and medical care in the US health care system is growing. ${ }^{1}$ This is especially true in Bureau of Primary Healthcare (BPHC)-funded health centers, which disproportionately serve patients whose health is affected by socioeconomic disadvantage. Little is known about the prevalence of social risks ${ }^{2}$ or social care interventions in BPHC settings. The current study examines the prevalence of social risk factors and social care among patients served in BPHCfunded health centers.

\section{METHODS}

We analyzed data from the 2014 Health Center Patient Survey (HCPS), a cross-sectional survey administered by the Health Resources and Services Administration (HRSA). The HCPS was conducted with a random convenience sample of patients from health centers receiving BPHC grants, including Community Health Centers (CHCs), Migrant Health Centers (MHCs), Health Care for the Homeless (HCH), and Public Housing Primary Care (PHPC) programs. Details of HCPS sampling scheme and administration are described elsewhere. $^{3,4}$ Survey data were merged with annually reported 2014 Uniform Data System (UDS) data, which include health center-reported characteristics. ${ }^{5}$

HCPS asked multiple questions related to patients' social risks, including employment status, education level, housing stability, and household income. Patients were also asked if they had ever received social care, including assistance from their health center to obtain free medications, access medical transportation, apply for government benefits, or access other basic needs resources (e.g., housing, employment, childcare, food, or clothing/shoes). We examined patient- and health center-level predictors of both social risk and social assistance. Predictor variables from HCPS included patient sociodemographics, health status, health care utilization, clinic urbanicity, and grantee type. Health center-level predictors from UDS included proportion of total staff FTE dedicated to enabling services (non-clinical services to improve health care access and outcomes), ${ }^{6}$ panel size, and regional location.

Weighted frequencies of patient demographics, health, and social risk factors, as well as health center-reported characteristics, were calculated in the full sample, then again in only those reporting receiving assistance, stratifying by type of assistance received. Chi-squared tests were used to compare differences between stratified samples. All data analyses were conducted using Stata/SE 15.0.

\section{RESULTS}

Of the 7002 survey respondents, 4225 (60.3\%) adult patients from 167 health centers had recorded responses to the measures of social risk factors or key study demographic variables and were included in the final study sample. Respondentreported demographics, health status and health care utilization, social risk factors and social care, and health center-level characteristics are shown in Table 1. Assistance with different types of social care varied by patient and health center characteristics (Table 2).

\section{DISCUSSION}

This study is the first to evaluate the prevalence of patientreported social risk factors and delivery of social care in a nationally representative sample of federally funded health centers. The population surveyed reported relatively high levels of social risks and low levels of assistance. Patients 
Table 1 Descriptors of Patient Respondents to 2014 Health Center Patient Survey $(n=4225$ Patients) and Matched Bureau of Primary Health Care-Funded Health Center Characteristics $(n=167$ Health Centers)

\begin{tabular}{|c|c|c|}
\hline & \multicolumn{2}{|l|}{ Descriptors } \\
\hline & Unweighted $(n)$ & Weighted (\%) $(95 \% \mathrm{CI})$ \\
\hline \multicolumn{3}{|l|}{ Patient-reported demographics ${ }^{\mathrm{a}}$} \\
\hline Gender : Female & 2676 & $64.9(60.4$ to 69.2$)$ \\
\hline \multicolumn{3}{|l|}{ Race/ethnicity } \\
\hline Non-Hispanic White & 1147 & $51.0(43.2$ to 58.7$)$ \\
\hline Non-Hispanic Black & 1095 & $21.2(16.3$ to 27.0$)$ \\
\hline Non-Hispanic Asian & 234 & $1.67(0.87$ to 3.18$)$ \\
\hline Other $^{\mathrm{c}} \mathrm{i}$ & 173 & $2.64(1.49$ to 4.65$)$ \\
\hline Hispanic & 1576 & $23.6(18.1$ to 30.2$)$ \\
\hline \multicolumn{3}{|l|}{ Age } \\
\hline $18-45$ & 1542 & $48.0(43.0$ to 54.1$)$ \\
\hline $45-65$ & 2230 & 39.4 (33.7 to 45.4$)$ \\
\hline $65+$ & 453 & $12.6(6.84$ to 22.1$)$ \\
\hline Recent immigrant $^{\mathrm{d}}$ & 1867 & $28.4(22.9$ to 34.7$)$ \\
\hline \multicolumn{3}{|l|}{ Insurance } \\
\hline Uninsured & 1024 & $29.3(22.5$ to 37.2$)$ \\
\hline Public & 1741 & $41.2(34.0$ to 48.9$)$ \\
\hline Private & 1460 & $29.5(23.1$ to 36.8$)$ \\
\hline \multicolumn{3}{|l|}{ Patient-reported health status/utilization ${ }^{\mathrm{a}}$} \\
\hline \multicolumn{3}{|l|}{ Reported health status } \\
\hline Excellent/very good/good & 2057 & $55.9(52.3$ to 59.5$)$ \\
\hline Fair/poor & 2168 & $44.1(40.5$ to 47.7$)$ \\
\hline Multiple chronic conditions ${ }^{\mathrm{e}}$ & 2262 & $51.4(46.9$ to 55.8$)$ \\
\hline Functional deficits ${ }^{\mathrm{f}}$ & 1151 & $26.8(23.3$ to 30.7$)$ \\
\hline Serious mental illness ${ }^{\mathrm{g}}$ & 595 & $12.7(9.83$ to 16.2$)$ \\
\hline Significant emotional distress ${ }^{\mathrm{h}}$ & 705 & $15.0(12.3$ to 18.1$)$ \\
\hline Lifetime drug use $\mathrm{e}^{\mathrm{i}}$ & 1855 & $46.3(39.4$ to 53.4$)$ \\
\hline \multicolumn{3}{|l|}{ Continuity with health center } \\
\hline$<6$ months & 507 & $9.87(7.73$ to 12.5$)$ \\
\hline 6 months to $1-3$ years & 1257 & 33.8 (29.5 to 38.3$)$ \\
\hline$>3$ years, $<5$ years & 590 & 14.5 (11.9 to 17.6$)$ \\
\hline $5+$ years & 1869 & $41.8(36.1$ to 47.7$)$ \\
\hline ED utilization last 12 months $^{\mathrm{j}}$ & 2150 & 59.8 (45.6 to 54.0$)$ \\
\hline Delay in medical care $\mathrm{k}^{\mathrm{k}}$ & 1655 & 40.7 (35.5 to 46.0$)$ \\
\hline Unable to access medical care ${ }^{\mathrm{k}}$ & 1574 & $39.3(34.2$ to 44.7$)$ \\
\hline Delay in prescription access ${ }^{1}$ & 1540 & $35.8(30.9$ to 41.0$)$ \\
\hline Unable to access prescription ${ }^{1}$ & 1369 & $33.0(28.8$ to 37.4$)$ \\
\hline \multicolumn{3}{|l|}{ Health center-reported practice level variables } \\
\hline \multicolumn{3}{|l|}{ State/region ${ }^{\mathrm{m}}$} \\
\hline Midwest & 584 & $20.9(12.0$ to 34.0$)$ \\
\hline North East & 720 & 19.7 (11.9 to 30.8$)$ \\
\hline South & 1208 & $30.5(19.4$ to 44.3$)$ \\
\hline West & 1713 & $28.8(18.1$ to 42.6$)$ \\
\hline Urbanicity $^{\mathrm{a}}$ & 3015 & $47.3(34.7$ to 60.1$)$ \\
\hline \multicolumn{3}{|l|}{ Health center grantee type ${ }^{a}$} \\
\hline Public Housing Primary Care (PHPC) & 365 & $0.99(0.68$ to 1.44$)$ \\
\hline Migrant Health Center (MHC) & 590 & $3.14(2.08$ to 4.73$)$ \\
\hline Health Care for the Homeless $(\mathrm{HCH})$ & 895 & 3.29 (2.23 to 4.82$)$ \\
\hline Community Health Center (CHC) & 2375 & $92.6(90.4$ to 94.3$)$ \\
\hline \multicolumn{3}{|l|}{ Health center panel size ${ }^{\mathrm{m}}$} \\
\hline$\leq 10 \mathrm{k}$ & 583 & $16.1(8.5$ to 28.3$)$ \\
\hline$>10 \mathrm{k}$ to $\leq 40 \mathrm{k}$ & 1912 & $51.2(38.4$ to 63.9$)$ \\
\hline$>40 \mathrm{k}$ & 1730 & $32.7(23.1$ to 44.0$)$ \\
\hline Enabling services staffing $<10 \% \mathrm{FTE}^{\mathrm{m}, \mathrm{n}}$ & 1834 & 51.7 (38.6 to 64.5$)$ \\
\hline \multicolumn{3}{|l|}{ Patient-reported social risk factors ${ }^{\mathrm{a}}$} \\
\hline Unstable employment $^{\circ}$ & 3104 & 70.3 (64.8 to 75.2$)$ \\
\hline Unstable housing $^{\mathrm{p}}$ & 1866 & 32.1 (27.6. to 36.9$)$ \\
\hline Education: $<$ high school diploma/GED ${ }^{\mathrm{q}}$ & 1869 & $34.7(30.4$ to 39.2$)$ \\
\hline$\%$ Federal poverty level (FPL) ${ }^{\mathrm{r}}$ & & \\
\hline$\leq 100$ & 2732 & $57.9(53.3$ to 62.3$)$ \\
\hline$\overline{1} 01-199$ & 1224 & 32.0 (28.1 to 36.2$)$ \\
\hline$\geq 200$ & 269 & $10.1(6.98$ to 14.4$)$ \\
\hline Patient report of assistance ${ }^{a}$ & & \\
\hline Obtaining free medications & 1407 & $28.0(23.5$ to 33.1$)$ \\
\hline Transportation to health center & 774 & $11.6(8.27$ to 16.1$)$ \\
\hline Applying for government benefits & 1177 & $23.7(19.5$ to 28.5$)$ \\
\hline Any basic needs & 620 & $8.46(6.24$ to 11.4$)$ \\
\hline Food & 439 & $5.68(4.15$ to 7.74$)$ \\
\hline Housing & 296 & $2.57(1.71$ to 3.86$)$ \\
\hline
\end{tabular}


Table 1. (continued)

\begin{tabular}{lll}
\hline \hline & \multicolumn{2}{l}{ Descriptors } \\
\cline { 2 - 3 } & Unweighted (n) & Weighted (\%) (95\% CI) \\
\hline Employment & 212 & $3.68(2.38$ to 5.67$)$ \\
Childcare & 61 & $1.22(0.691$ to 2.16$)$ \\
Clothing/shoes & 309 & $2.99(1.88$ to 4.72$)$ \\
\hline
\end{tabular}

${ }^{a}$ From 2014 Health Center Patient Survey (HCPS) dataset

${ }^{b}$ Gender (biological sex at birth)

${ }^{c}$ Non-Hispanic other race (patients who selected multiple races or a specific race group with too few respondents to maintain confidentiality)

${ }^{d}$ Recent immigration (speaking non-English language at home and/or being born outside of the USA)

${ }^{e}$ Multiple chronic conditions $\geq 2$ reported diagnoses of asthma, cancer, kidney disease, COPD, DM, CHF, hepatitis B or C, HIV, HLD, HTN, ischemic heart disease, stroke)

${ }^{f}$ Functional impairment $(\geq 1$ difficulty with activities of daily living (ADLs))

${ }^{g}$ Serious mental illness (reported diagnosis of schizophrenia or bipolar disorder)

${ }^{h}$ High psychological distress in the past 30 days (score $\geq 13$ on Kessler Psychological Distress Scale)

${ }^{i}$ Lifetime drug use: WHO Alcohol, Smoking and Substance Involvement Screening Test (ASSIST)

${ }^{j}$ One or more emergency department (ED) visits in past year

${ }^{k}$ Needing medical care in the past year but experiencing delay or unmet needs

${ }^{l}$ Needing prescription meds in the past year but experiencing delay or unmet needs

${ }^{m}$ From 2014 Uniform Data System

${ }^{n}$ Proportion of full-time equivalents (FTEs) designated as enabling services, per UDS reporting guidelines. Absolute numbers were unavailable given the need to maintain health center anonymity. Within enabling services staffing, individual types of staff included case managers, patient and community education specialists, outreach workers, transportation workers, eligibility assistance workers, interpretation staff, and personnel performing other enabling services activities

${ }^{\circ}$ Currently employed for pay vs. not

${ }^{p}$ Usually slept during the past week in either (1) in an emergency shelter, transitional shelter, or car, anywhere outside, or any other place not meant for habitation; (2) in a house, apartment, or room that they did not rent or own; (3) in their own place but moved two or more times in the past year or had been unable to pay the rent or mortgage at any time; (4) in their own place without multiple moves or difficulty paying rent/mortgage, but previously homeless versus stably housed without prior housing problems

$q_{\geq}$High school vs. not

${ }^{r}$ Measure of household income

Table 2 Percentage of Patients Who Reported Receiving Assistance from Their Bureau of Primary Health Care-Funded Health Center, by Patient Demographics, Self-Reported Health Status and Health Care Utilization, Practice-Level Characteristics, and Self-Reported Social Risk Factors

\begin{tabular}{|c|c|c|c|c|c|c|c|c|c|c|c|c|}
\hline & \multicolumn{12}{|c|}{ Type of reported assistance } \\
\hline & \multicolumn{3}{|c|}{ Obtaining free medications } & \multicolumn{3}{|c|}{$\begin{array}{l}\text { Health center } \\
\text { transportation }\end{array}$} & \multicolumn{3}{|c|}{$\begin{array}{l}\text { Applying to government } \\
\text { benefits }\end{array}$} & \multicolumn{3}{|c|}{ With basic needs* } \\
\hline & $\begin{array}{l}\text { Unwtd }_{(n)}^{\dagger} \\
\end{array}$ & $\begin{array}{l}\text { Wtdt } \\
(\%)\end{array}$ & $\begin{array}{l}p \\
\text { value }\end{array}$ & $\begin{array}{l}\text { Unwtd } \\
(n)\end{array}$ & $\begin{array}{l}\text { Wtd } \\
(\%)\end{array}$ & $\begin{array}{l}p \\
\text { value }\end{array}$ & $\begin{array}{l}\text { Unwtd } \\
(n)\end{array}$ & $\begin{array}{l}\text { Wtd } \\
(\%)\end{array}$ & $\begin{array}{l}p \\
\text { value }\end{array}$ & $\begin{array}{l}\text { Unwtd } \\
(n)\end{array}$ & $\begin{array}{l}\text { Wtd } \\
(\%)\end{array}$ & $\begin{array}{l}p \\
\text { value }\end{array}$ \\
\hline \multicolumn{13}{|c|}{ Patient-reported demographics } \\
\hline Gender & & & & & & & & & & & & \\
\hline Female & 624 & 31.7 & \multirow[t]{2}{*}{0.24} & 358 & 11.4 & \multirow[t]{2}{*}{0.92} & 751 & 23.6 & \multirow[t]{2}{*}{0.87} & 564 & 27.9 & \multirow[t]{2}{*}{0.90} \\
\hline Male & 783 & 26.0 & & 416 & 11.7 & & 426 & 24.0 & & 910 & 27.6 & \\
\hline Race/ethnicity & & & & & & & & & & & & \\
\hline $\begin{array}{l}\text { Non- } \\
\text { Hispanic White }\end{array}$ & 430 & 27.1 & \multirow[t]{6}{*}{0.32} & 191 & 6.3 & \multirow{3}{*}{$\begin{array}{l}< \\
0.01\end{array}$} & 287 & 18.9 & \multirow[t]{6}{*}{0.01} & 374 & 22.0 & \multirow[t]{6}{*}{0.02} \\
\hline Non-Hispanic & 446 & 29.4 & & 282 & 24.4 & & 283 & 28.8 & & 393 & 34.7 & \\
\hline $\begin{array}{l}\text { Black } \\
\text { Non- }\end{array}$ & & & & & & & & & & & & \\
\hline $\begin{array}{l}\text { Non- } \\
\text { Hispanic Asian }\end{array}$ & 63 & 21.9 & & 24 & 14.8 & & 97 & 39.9 & & 109 & 43.7 & \\
\hline Other & 63 & 4.5 & & 56 & 18.5 & & 51 & 15.1 & & 64 & 38.3 & \\
\hline Hispanic & 405 & 26.9 & & 221 & 10.4 & & 459 & 29.4 & & 534 & 31.4 & \\
\hline \multicolumn{13}{|l|}{ Age } \\
\hline $18-44$ & 413 & 24.5 & \multirow[t]{3}{*}{$<0.01$} & 239 & 11.3 & \multirow[t]{3}{*}{0.05} & 440 & 25.1 & \multirow{3}{*}{0.34} & 530 & 28.0 & \multirow{3}{*}{0.12} \\
\hline $45-65$ & 894 & 35.9 & & 481 & 13.9 & & 640 & 23.8 & & 830 & 30.1 & \\
\hline $65+$ & 100 & 17.1 & & 54 & 5.5 & & 97 & 16.3 & & 114 & 19.3 & \\
\hline \multicolumn{13}{|c|}{ Recent immigrant } \\
\hline Yes & 498 & 28.0 & \multirow[t]{2}{*}{0.97} & 255 & 10.8 & \multirow[t]{2}{*}{0.65} & 568 & 29.3 & \multirow[t]{2}{*}{0.04} & 661 & 31.2 & \multirow[t]{2}{*}{0.241} \\
\hline No & 909 & 28.1 & & 519 & 11.9 & & 609 & 21.5 & & 813 & 26.3 & \\
\hline Insurance & & & & & & & & & & & & \\
\hline Public & 588 & 35.8 & 0.01 & 365 & 18.5 & & 566 & 28.5 & 0.12 & 693 & 32.0 & 0.53 \\
\hline
\end{tabular}


Table 2. (continued)

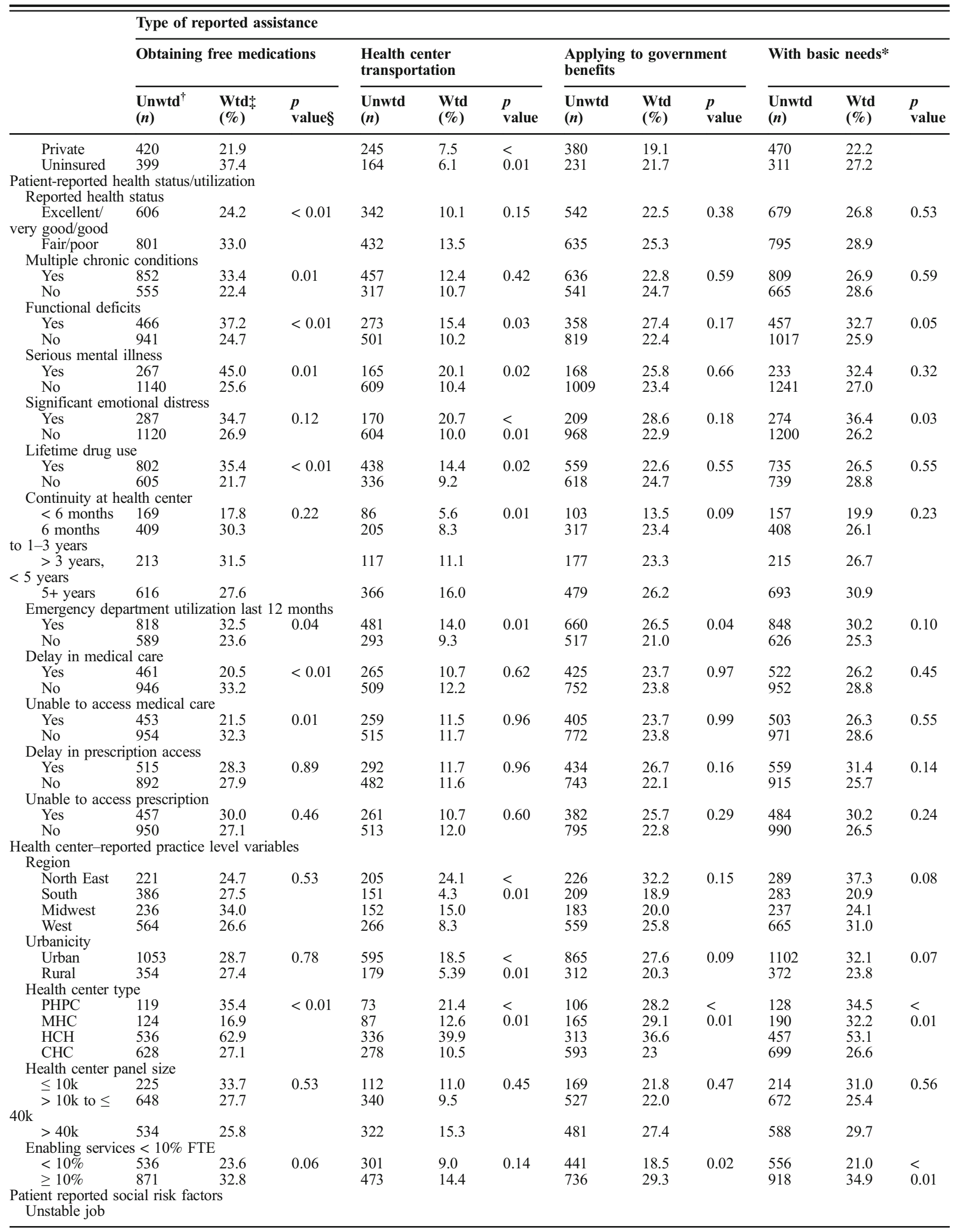


Table 2. (continued)

\begin{tabular}{|c|c|c|c|c|c|c|c|c|c|c|c|c|}
\hline & \multicolumn{12}{|c|}{ Type of reported assistance } \\
\hline & \multicolumn{3}{|c|}{ Obtaining free medications } & \multicolumn{3}{|c|}{$\begin{array}{l}\text { Health center } \\
\text { transportation }\end{array}$} & \multicolumn{3}{|c|}{$\begin{array}{l}\text { Applying to government } \\
\text { benefits }\end{array}$} & \multicolumn{3}{|c|}{ With basic needs* } \\
\hline & $\begin{array}{l}\text { Unwtd }_{(n)}^{\dagger} \\
\text { (n) }\end{array}$ & $\begin{array}{l}\text { Wtd } \\
(\%)\end{array}$ & $\begin{array}{l}p \\
\text { value§ }\end{array}$ & $\begin{array}{l}\text { Unwtd } \\
(n)\end{array}$ & $\begin{array}{l}\text { Wtd } \\
(\%)\end{array}$ & $\begin{array}{l}p \\
\text { value }\end{array}$ & $\begin{array}{l}\text { Unwtd } \\
(n)\end{array}$ & $\begin{array}{l}\text { Wtd } \\
(\%)\end{array}$ & $\begin{array}{l}p \\
\text { value }\end{array}$ & $\begin{array}{l}\text { Unwtd } \\
(n)\end{array}$ & $\begin{array}{l}\text { Wtd } \\
(\%)\end{array}$ & $\begin{array}{l}p \\
\text { value }\end{array}$ \\
\hline Yes & 1103 & 28.0 & 0.96 & 646 & 12.6 & 0.21 & 886 & 23.8 & 0.98 & 1126 & 27.4 & 0.75 \\
\hline No & 304 & 28.2 & & 128 & 9.2 & & 291 & 23.7 & & 348 & 28.6 & \\
\hline \multicolumn{13}{|c|}{ Unstable housing } \\
\hline Yes & 858 & 38.5 & $<0.01$ & 493 & 16.4 & $<$ & 575 & 26.1 & 0.35 & 793 & 33.0 & 0.04 \\
\hline No & 549 & 23.1 & & 281 & 9.4 & 0.01 & 602 & 22.6 & & 681 & 25.2 & \\
\hline \multicolumn{13}{|c|}{ Education: < high school diploma/ GED } \\
\hline Yes & 592 & 26.7 & 0.52 & 359 & 13.2 & 0.32 & 518 & 24.5 & 0.77 & 647 & 28.4 & 0.80 \\
\hline No & 815 & 28.8 & & 415 & 10.8 & & 659 & 23.3 & & 827 & 27.4 & \\
\hline \multicolumn{13}{|c|}{$\%$ Federal poverty level (FPL) } \\
\hline$\leq 100$ & 1002 & 27.7 & 0.13 & 587 & 14.3 & 0.05 & 794 & 25.9 & 0.01 & 1026 & 30.9 & $<$ \\
\hline $101-199$ & 338 & 31.6 & & 155 & 7.3 & & 325 & 23.9 & & 383 & 27.0 & 0.01 \\
\hline$\geq 200$ & 67 & 18.5 & & 32 & 10.1 & & 58 & 10.4 & & 65 & 11.5 & \\
\hline
\end{tabular}

*Basic needs assistance with housing, job, food, childcare, clothing/shoes

Untd unweighted

${ }^{7}$ Wtd weighted

${ }^{\S}$ Two-tailed $p$ values obtained from weighted chi-square analyses comparing differences in patients who reported receiving assistance from their health center vs. those who did not report assistance. When variables have multiple categories, $p$ values reflect between-group differences across all categories

primarily reported receiving assistance with health care access and other medical care-related social risks, like transportation. Fewer patients reported assistance around basic material needs like food or housing. Receiving assistance varied by sociodemographic, health care utilization, and health center characteristics. In general, higher proportions of patients with poorer health status reported assistance accessing medical transportation and obtaining free medications. At the health center level, patients of clinics with more enabling services staffing reported receiving more assistance with basic material needs resources.

The HCPS survey design should influence the interpretation of these findings. The cross-sectional survey relied entirely on patient-reported health indicators. Questions about assistance were not temporally bounded (e.g., "Have you ever received assistance with..."), nor did they have a corresponding question about whether the patient perceived a need for assistance. Additionally, questions about applying for benefits were ambiguous, but likely mostly pertained to enrolling in health insurance. Despite these limitations, the survey is a useful indicator of the prevalence of social risk factors and delivery of social care in federally funded health centers. We found that delivery of social care is uneven across health center settings, driven both by patient- and health center-level variables. This may suggest that equally complex patients may receive different services in different settings. As interest grows around integrated social and medical care delivery, policy innovations can use data about the prevalence of social risk and availability of social care initiatives to improve comprehensive health programs.

Acknowledgments: We would like to thank the Data Analytics Team within the Data and Evaluation Division in the Bureau of Primary Health Care at HRSA for their support.
Corresponding Author: Emilia De Marchis, MD, MAS; Department of Family \& Community Medicine University of California, San Francisco, 3333 California Street, Suite 465, San Francisco, CA 94118, USA (e-mail: Emilia.Demarchis@ucsf.edu).

Funding Information This publication was supported by the Robert Wood Johnson Foundation (RWJF) and a fellowship training grant by the National Research Service Award (NRSA) T32HP19025. Its contents are solely the responsibility of the authors and do not represent the official views of the RWJF or NRSA.

\section{Compliance with Ethical Standards:}

Conflict of Interest: The authors declare that they do not have a conflict of interest.

\section{REFERENCES}

1. National Academies of Sciences Engineering and Medicine. Integrating Social Needs Care into the Delivery of Health Care to Improve the Nation's Health. Available at: http://nationalacademies.org/hmd/activities/healthservices/ integratingsocialneedscareintothedeliveryofhealthcaretoimprovethenationshealth.aspx. Accessed August 21, 2019.

2. Alderwick H, Gottlieb LM. Meanings and Misunderstandings: A Social Determinants of Health Lexicon for Health Care Systems. Milbank $Q$ 2019;97(2):407-419.

3. Baggett TP, Berkowitz SA, Fung V, Gaeta JM. Prevalence of Housing Problems Among Community Health Center Patients. JAMA 2018;319(7):717-719.

4. Health Resources and Services Administration. 2014 Health Center Patient Survey Data File User's Manual. Available at: https://bphc.hrsa. gov/datareporting/research/hcpsurvey/2014usermanual.pdf. Accessed August 21, 2019.

5. Bureau of Primary Health Care. National Health Center Data. Available at: https://bphc.hrsa.gov/uds/datacenter.aspx. Accessed August 21, 2019.

6. Weir RC, Proser M. Highlighting the Role of Enabling Services at Community Health Centers: Collecting Data to Support Service Expansion \& Enhanced Funding. Available at: http://www.nachc.org/wp-content/uploads/2015/ 06/EnablingServicesReport.pdf. Accessed August 21, 2019.

Publisher's Note Springer Nature remains neutral with regard to jurisdictional claims in published maps and institutional affiliations. 